\title{
Capacity Based Adaptive Power Allocation for the OFDM Relay Networks with Limited Feedback
}

\author{
Yong Liu*, Wen Chen*, and Xiaopeng Huang ${ }^{\dagger}$, \\ * Wireless Network Transmission Laboratory, Shanghai Jiao Tong University, Shanghai, China, 200240 \\ Email: \{yongliu1982, wenchen\}@sjtu.edu.cn \\ ${ }^{\dagger}$ Electrical and Computer engineering department, Stevens Institute of Technology, New Jersey, USA \\ Email: xhuang3@stevens.edu
}

\begin{abstract}
In this paper, we propose a dynamic power allocation scheme for a single-relay network based on orthogonal frequency division multiplexing (OFDM) modulation with limited feedback. We consider an Amplify-and-Forward (AF) cooperative diversity model, where one source node communicates with one destination node assisted by one half duplex relay. The power allocation scheme employs a codebook of quantized power allocation vectors designed offline equipped on the source, relay, and destination. The destination, who has full knowledge of channel state information (CSI), chooses the best power allocation vector and sends back its index to the source and relay nodes, which results in a dramatic reduced overhead over conventional resource allocation. First we achieve the optimal power allocation solution as the function of channel realizations with maximum capacity. Then we present an adaptation of the Lloyd algorithm to construct a codebook to quantize the optimal power allocation vectors subject to the amount of feedback. Simulations show that a negligible performance loss could be achieved with just a few feedback bits at different levels of SNRs.
\end{abstract}

Index Terms-Limited Feedback, OFDM, Power Allocation, Amplify-and-Forward, Lloyd Algorithm.

\section{INTRODUCTION}

Considering the power, hardware complexity and cost limitation of wireless terminals, cooperative relaying has recently emerged as an effective way to exploit spatial diversity, combat wireless channel fading and entend coverage without antenna arrays [1]-[3]. For example, IEEE 802.16 is currently integrating relays for multihop communication [4]. Two main relay strategies have been adopted in such scenarios: Amplify-andForward (AF) and Decode-and-Forward (DF). In AF scheme, the received signal is amplified and then retransmitted by the relay without performing decoding, while the latter decodes the received signal and re-encodes it for retransmission.

OFDM is a mature technique to mitigate the problems of frequency of selectivity and inter-symbol interference [5]. By employing IFFT and FFT processing, a frequency-selective fading channel can be converted into parallel flat-fading ones. Due to its inherent robustness against frequency-selective fading and its potential for high spectral efficiency, OFDM has been proposed as a most promising candidate for the physical layer in 4G cellular system [5]. The combination of cooperative relaying with OFDM offers an even more promising perspective in increasing cell coverage and system capacity.
Power efficiency is a critical design criteria in wireless networks due to the limited budget of transmit power at transmitters. Power allocation algorithm has been extensively studied in the context of both single-carrier and multi-carrier relaying channels [6]-[14]. In [6], we consider a DF cooperative model and propose a power allocation strategy with quantized CSI feedback in single-carrier case. In [7], Ahmed et al. propose resource control algorithms for DF relaying. Then he studies a single relay system with AF relaying over frequency-flat Rayleigh channels, and solves the power allocation problem aiming to minimize the outage probability in [8]. Similarly, the optimal power allocation that maximizes the system capacity between single antenna source and relay was proposed in [9]. On the other hand, the optimal power allocation at source and relay in a two-hop non-regenerative relaying scheme with OFDM modulation is investigated in [10]. In [11], the same authors propose the optimum power allocation to maximize the instantaneous rate of OFDM relay system in frequency-selective channels. Unlike AF relaying in [11], Ying et al. [12] work on the similar problem but for DF relaying OFDM systems. In [13], Ma et al. introduce bit and power loading algorithms to minimize the transmit power consumption for $\mathrm{AF}$ and selected $\mathrm{DF}$ modes at a target throughput.

It is well known that resource allocation employing channel adaptive signaling can yield significant improvement in almost any performance metric of relay channels [6],[14]-[16]. The tremendous innovation that realizes instantaneous channel adaptation practice is the use of feedback whose history may trace back to Shannon [17]. It has been proven that the error and capacity performance with perfect channel information at the transmitter is significantly better compared to the case that no CSI can be used [18]-[19]. However, the increase of performance came at the price of obtaining the full knowledge of CSI at the transmitters. Obviously this needs too much extra spectral resource. Some research have been done to achieve this in a system with limited feedback. One can either send back a quantized version of CSI or send the quantized power allocation vectors [8], or even just sends back the index of the best vector in a codebook of power allocation vectors shared by all the nodes [20], [21]. Most of these works are studied in the context of MIMO and OFDM systems. To the best of our knowledge, using limited feedback in a relay OFDM system 
has not been well-exploited yet.

This work is based on our previous work [6] by extending single-carrier relaying channels to the multi-carrier one. In this paper, we present a power allocation protocol for an OFDM based cooperative model under different levels of quantized CSI feedback. In our feedback scheme, we propose the codebook design for maximum capacity metrics with a joint power constraint, and employ the Lloyd algorithm to construct the optimal codebook. An intensive performance analysis is provided for the proposed scheme. The conventional power allocation algorithm is compared with the proposed limitedfeedback power controller. We also investigate the impact of location of relay node on the BER and system capacity. We find that the performance gap between the system capacities achieved by the proposed algorithm and the uniform allocation is larger when the channel conditions of $S \rightarrow R$ and $R \rightarrow D$ are quite different. We also find that only a few feedback bits are necessary to achieve most of the performance gains of the perfect feedback power allocation algorithm, and the loss becomes negligible when SNR is high enough.

The rest of this paper is organized as follows. In Section II, we briefly describe the system model. In Section III, we evaluate the error rate and capacity for the relay system, and propose the power allocation solution based on the two criterions with limited feedback. In Section IV, we compare the performance of the proposed scheme to perfect and no CSI cases and present some simulation results. The conclusions are drawn in section $\mathrm{V}$.

\section{SySTEM MODEL}

In this paper, we consider a time division duplex OFDM cooperative diversity model where one source node communicates with one destination assisted by one half duplex relay. The relay system is depicted in Fig. 1, whose relay does not produce its own data and only assists in the communication between the source and the destination. It is assumed that a total $B$ bandwidth is divided into $N$ independent sub-channels. Communication between the source and destination takes place in two phases, i.e., the listening phase and the relaying phase. In the listening phase, the source broadcasts its information through $N$ sub-carriers, the relay and the destination listen, while in the relaying phase, the relay transmits an amplified version of the received signal on the same subcarrier. Finally the destination tries to decode based on all the signals received during both phases.

We assume that channels experienced by the $n$th subcarrier of source-destination, source-relay and relay-destination in the system are attenuated by fading coefficient $h_{S D}^{n}, h_{S R}^{n}$ and $h_{R D}^{n}$ respectively. Then the received signal at the listening phase are

$$
\begin{aligned}
& y_{d n}^{(1)}=h_{S D}^{n} * \sqrt{P_{s n}} * s_{n}+z_{d n}^{(1)}, \\
& y_{r n}=h_{S R}^{n} * \sqrt{P_{s n}} * s_{n}+z_{r n}
\end{aligned}
$$

where $y_{d n}^{(1)}$ and $y_{r n}$ are the received signal at the destination and relay nodes, $z_{d n}^{(1)} \sim \mathcal{C N}\left(0, \sigma_{d}\right)$ and $z_{r n} \sim \mathcal{C N}\left(0, \sigma_{r}\right)$ are the additive noises at the relay and the destination, respectively.

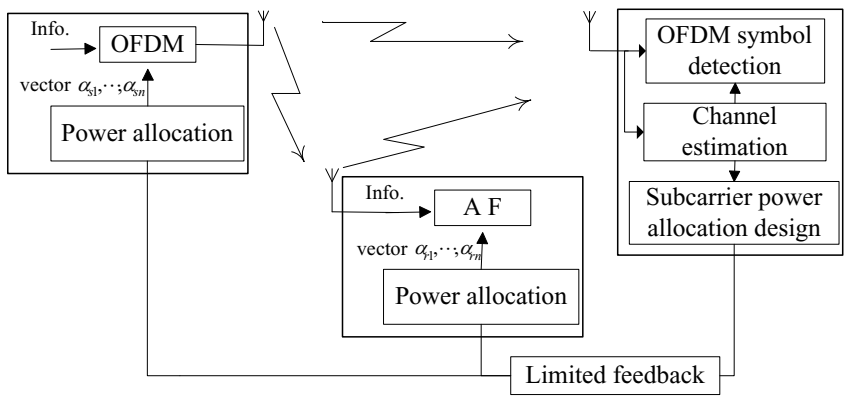

Fig. 1. System block diagram of OFDM cooperative diversity model with limited feedback

$\sqrt{P_{s n}}=\alpha_{s n} P_{t o t}$ is the $\alpha_{s n}$ portion of total power $P_{t o t}$. In the relaying phase, the received signal at the destination is

$$
y_{d n}^{(2)}=h_{R D}^{n} * \sqrt{\frac{P_{r n}}{P_{s n}\left|h_{S R}^{n}\right|^{2}+\sigma_{r}^{2}}} * y_{r n}+z_{d n}^{(2)},
$$

where $z_{d n}^{(2)} \sim \mathcal{C N}\left(\mu, \sigma_{d}^{2}\right)$ is the additive noise at the destination in the relaying phase. $\sqrt{P_{r n}}=\alpha_{r n} P_{t o t}$ is the $\alpha_{r n}$ portion of total power $P_{t o t}$. We call $\alpha_{\mathbf{s}}=\left[\alpha_{s 1}, \ldots, \alpha_{s N}\right]$ transmit $P A$ vector and $\alpha_{\mathbf{r}}=\left[\alpha_{r 1}, \ldots, \alpha_{r N}\right]$ relaying $P A$ vector. Those vectors have to be carefully designed to maximize the capacity.

Provided the AF relay works in time division duplex mode, we assume that the destination combines the signal received from the source and signal from the relay by maximal ratio combining (MRC). The final received SNR is composed of the received SNR in the two stages. For example, the two received SNRs at the $n$th subcarrier are given by

$$
S N R_{n}^{(1)}=\frac{\alpha_{s n} P_{t o t}\left|h_{S D}^{n}\right|^{2}}{\sigma_{d}^{2}},
$$

and

$$
S N R_{n}^{(2)}=\frac{\frac{\alpha_{s n} P_{t o t}\left|h_{S R}^{n}\right|^{2}}{\sigma_{r}^{2}} \cdot \frac{\alpha_{r n} P_{t o t}\left|h_{R D}^{n}\right|^{2}}{\sigma_{d}^{2}}}{1+\frac{\alpha_{s n} P_{t o t}\left|h_{S R}^{n}\right|^{2}}{\sigma_{r}^{2}}+\frac{\alpha_{r n} P_{t o t}\left|h_{R D}^{n}\right|^{2}}{\sigma_{d}^{2}}},
$$

respectively [1]. So the final received $\operatorname{SNR}$ at subcarrier $n$ is

$$
\begin{aligned}
S N R_{n}=\frac{\alpha_{s n} P_{t o t}\left|h_{S D}^{n}\right|^{2}}{\sigma_{d}^{2}} & \\
& +\frac{\frac{\alpha_{s n} P_{t o t}\left|h_{S R}^{n}\right|^{2}}{\sigma_{r}^{2}} \cdot \frac{\alpha_{r n} P_{t o t}\left|h_{R D}^{n}\right|^{2}}{\sigma_{d}^{2}}}{1+\frac{\alpha_{s n} P_{t o t}\left|h_{S R}^{n}\right|^{2}}{\sigma_{r}^{2}}+\frac{\alpha_{r n} P_{t o t}\left|h_{R D}^{n}\right|^{2}}{\sigma_{d}^{2}}} .
\end{aligned}
$$

Given channel realization, the capacity of an OFDM single AF relay system is

$$
C=\frac{1}{2} \sum_{n=1}^{N} \log _{2}\left(1+S N R_{n}\right) .
$$

\section{Optimal Power Allocation with Limited FEEDBACK}

In this section, we present optimal power allocation algorithms based on maximum capacity with limited channel state information (CSI) feedback. 


\section{A. General Procedure}

If the source and relay nodes have full knowledge of CSI, the power allocation vectors $\alpha_{\mathbf{s}}$ and $\alpha_{\mathbf{r}}$ can be easily determined. However, the perfect CSI is not available at the source/relay. Thus a quantized vector is fed back to source/relay through a limited number of feedback bits. Denote $\left\{\mathbf{h}_{i}, i \in\{S D, S R, R D\}\right\}$ as the state of the subcarriers at a particular time instant. Then the CSI can be defined by a 3 -tuple vector $\mathbf{h}=\left(\mathbf{h}_{S D}, \mathbf{h}_{S R}, \mathbf{h}_{R D}\right)$. Suppose that the destination has a perfect CSI. Then it would be quantized at first. If the destination node carry out $K$ bits feedback, the CSI space will be quantized into $H=2^{K}$ regions. We will adopt Llyod algorithm [22] to quantize the CSI space. The destination, upon the current CSI, selects a power vector $\mathbf{A}_{q}=\left(\alpha_{s, q}, \alpha_{r, q}\right)$ from the designed codebook $\mathbb{C}=\left\{\left(\alpha_{s}, \alpha_{r}\right)_{1}, \ldots,\left(\alpha_{s}, \alpha_{r}\right)_{H}\right\}$ whose size is $H$. Then it broadcasts the index $q$ to both the source and relay. We assume that the source/relay have also been equipped with the same codebook. Upon the received index $q$, the source transmits with power allocation vector $\alpha_{s, q}$ and the relay with power $\alpha_{r, q}$. The element of the codebook is designed to maintain the joint power constraints of the source and relay, i.e., elements of each power allocation vector should ensure that the total power budget to allocate to the source and relay is $P_{t o t}$,

$$
\mathbf{1}^{T} \alpha_{s}+\mathbf{1}^{T} \alpha_{r}=1
$$

Then the problem lies in the design of the codebook, that is power control mapping, $\mathbf{F}: \mathfrak{R} \rightarrow \mathbb{C}$, where $\mathfrak{R}$ is the instantaneous CSI space, with the average power constraint $\mathbf{1}^{T} \alpha_{s}+\mathbf{1}^{T} \alpha_{r}=1$. Thereby, the essence of our algorithm is to realize the objective to find an optimal $\mathbf{F}$ in order to optimize some performance criteria while meeting the power constraint. We will employ the Lloyd algorithm with a modified distortion function to design the codebook.

\section{B. Lloyd Algorithm Based Codebook Design}

The key points of Lloyd algorithm are centroid rule and nearest rule [22]. They are iteratively used in our codebook optimization. Actually the centroid rule aims to select the optimal codeword to maximize the system capacity in a region, while the latter is designed to determine the region in which the vectors in this region are closest to the codeword of this region.

The crucial point in the algorithm is the design of average distortion function [22], different performance criteria may have different type of distortion function. Take optimizing capacity performance criteria for example, we use the error distance function to measure the average distortion.

Given the capacity of the system as (6), the optimization issue becomes

$$
\begin{gathered}
\max _{A} C\left(\alpha_{s}, \alpha_{r} \mid P_{t o t}\right) \\
\text { s.t. } \mathbf{1}^{T} \alpha_{s}+\mathbf{1}^{T} \alpha_{r}=1, \\
\alpha_{s} \geq 0, \alpha_{r} \geq 0
\end{gathered}
$$

The codebook size is $H=2^{K}$, and we have to produce training vectors $\left(T_{i} \mid i=0,1, \ldots, M\right)$ with $M \gg H$. Then the algorithm can be expressed as follows.

A: Set $i=1$, and randomly select the initial codebook $\left\{\mathbb{C}^{0}=\left\{A_{1}^{0}, \ldots, A_{H}^{0}\right\}\right.$ from the training vectors $\left(T_{i} \mid i=\right.$ $0,1, \ldots, M)$;

B: Cluster the training vectors into $H$ groups as

$$
R_{q}=\left\{T_{i} \mid C\left(T_{i}, R_{q}\right)>C\left(T_{i}, R_{p}\right) \forall p \neq q\right\}
$$

for $q=1,2, \ldots, H$.

C: Construct a new codebook $\mathbb{C}^{i}$ with power allocation vector $A_{k}^{i}$ defined as

$$
\begin{aligned}
A_{k}^{i} & =\arg \max _{A} \mathbf{E}_{T \in R_{q}}\left(C\left(A \mid P_{\text {tot }}\right)\right) \\
& =\arg \max _{A} \mathbf{E}_{T \in R_{q}}\left(\frac{1}{2} \sum_{n=1}^{N} \log _{2}\left(1+S N R_{n}\right)\right) .
\end{aligned}
$$

D: We define the average distortion function $D(\mathbb{C})$ as

$$
D(\mathbb{C})=E_{T}\left(C\left(A_{\text {opt }} \mid h\right)-\max _{0 \leq i \leq H} C\left(A_{i} \mid h\right)\right),
$$

if $D\left(\mathbb{C}_{i}\right)<D\left(\mathbb{C}_{i-1}\right)+\varepsilon$, stop iteration and set $\mathbb{C}^{*}=$ $\mathbb{C}_{i-1}$. Otherwise set $i=i+1$ and go back to step $B$.

Using the centroid rule and the nearest neighbor rule iteratively, the system capacity will increases monotonically. Actually, when Lloyd algorithm is terminated, the destination has just searched over all codewords in the codebook and selects the best power allocation vector with maximum capacity. It just needs to send back $B=\log _{2} H$ bits representing the codeword index to the source and relay nodes.

\section{Power Allocation Design with Capacity Criteria}

As shown in (6) and (8), the optimization issue can be formulated as

$$
\begin{aligned}
\max _{A} \frac{1}{2} \sum_{n=1}^{N} \log _{2}\left(1+\frac{\alpha_{s n} P_{t o t}\left|h_{S D}^{n}\right|^{2}}{\sigma_{d}^{2}}\right. & \\
& \left.+\frac{\frac{\alpha_{s n} P_{t o t}\left|h_{S R}^{n}\right|^{2}}{\sigma_{r}^{2}} \cdot \frac{\alpha_{r n} P_{t o t}\left|h_{R D}^{n}\right|^{2}}{\sigma_{d}^{2}}}{1+\frac{\alpha_{s n} P_{t o t}\left|h_{S R}^{n}\right|^{2}}{\sigma_{r}^{2}}+\frac{\alpha_{r n} P_{t o t}\left|h_{R D}^{n}\right|^{2}}{\sigma_{d}^{2}}}\right), \\
\text { s.t. } & \mathbf{1}^{T} \alpha_{s}+\mathbf{1}^{T} \alpha_{r}=1, \\
& \alpha_{s} \geq 0, \alpha_{r} \geq 0,
\end{aligned}
$$

In this paper, the optimal power allocation vector $A$ that maximizes $C\left(A \mid P_{t o t}\right)$ is achieved by the Lagrange method. 
Make the cost function

$$
\begin{aligned}
L(A, \lambda)= & L\left(\left(\alpha_{s}, \alpha_{s}\right), \lambda\right) \\
= & \sum_{n=1}^{N} \log _{2}\left(1+\alpha_{s n} \rho_{S D}+\frac{\alpha_{s n} \rho_{S R} \cdot \alpha_{r n} \rho_{R D}}{1+\alpha_{s n} \rho_{S R}+\alpha_{r n} \rho_{R D}}\right) \\
& -\lambda\left(\sum_{n=1}^{N}\left(\alpha_{s n}+\alpha_{r n}\right)-1\right)
\end{aligned}
$$

where

$$
\begin{aligned}
\rho_{S D} & =\frac{P_{t o t}\left|h_{S D}^{n}\right|^{2}}{\sigma_{d}^{2}}, \\
\rho_{S R} & =\frac{P_{t o t}\left|h_{S R}^{n}\right|^{2}}{\sigma_{r}^{2}}, \\
\rho_{R D} & =\frac{P_{t o t}\left|h_{R D}^{n}\right|^{2}}{\sigma_{d}^{2}},
\end{aligned}
$$

and $\lambda$ denotes the Lagrange multiplier. Then

$$
\lambda=\frac{\partial L(A, \lambda)}{\alpha_{s}}=\frac{\partial L(A, \lambda)}{\alpha_{r}} .
$$

Take derivatives of $L$ with respect to $\alpha_{s n}$ and $\alpha_{r n}$ respectively. We have

$$
\begin{aligned}
& \alpha_{s n} \rho_{S R}= \\
& \quad \alpha_{r n} \rho_{R D} \frac{1+\rho_{S D}+\sqrt{1+\rho_{S D} \rho_{R D}+\rho_{S R}\left(\rho_{R D}-\rho_{S D}\right)}}{\rho_{R D}-\rho_{S D}} .
\end{aligned}
$$

Take derivatives of $L$ with respect to $\lambda$. We have

$$
0=\frac{\partial L(A, \lambda)}{\lambda} \Longleftrightarrow \sum_{n=1}^{N}\left(\alpha_{s n}+\alpha_{r n}\right)=1 \text {. }
$$

Considering (14), (15) and (16) together, we can obtain the following optimal solution

$$
\left\{\begin{aligned}
\alpha_{r n} & =\left[\frac{1}{\lambda}-\frac{1}{\rho_{R D}}(1+\psi)^{2}\right]^{+} \frac{\psi}{\psi+\psi^{2}+\frac{\rho_{S D}}{\rho_{S R}}(\psi+1)^{2}}, \\
\alpha_{s n} & =\frac{\rho_{S R}}{\rho_{S D}} \cdot \alpha_{r n} .
\end{aligned}\right.
$$

where

$$
\psi=\frac{\rho_{R D}-\rho_{S D}}{1+\rho_{S D}+\sqrt{1+\rho_{S D} \rho_{R D}+\rho_{S R}\left(\rho_{R D}-\rho_{S D}\right)}},
$$

and

$$
[x]^{+}= \begin{cases}x & : \text { when } 0<x<\frac{1}{N}, \\ 0 & : \text { otherwise. }\end{cases}
$$

By now, we have found a pair of optimal power vector $\left(\alpha_{s}, \alpha_{r}\right)$ maximizing the system capacity. Our analysis can be further simplified if we assume that the received SNR defined in (3) is high enough, that is $\rho_{i} \gg 1$. Then the capacity in (6) can be further approximated as

$$
\mathbf{C}\left(A_{\text {opt }} \mid h\right)=\frac{1}{2} \sum_{n=1}^{N} \log _{2}\left(\alpha_{s n} \rho_{S D}+\frac{\alpha_{s n} \rho_{S R} \cdot \alpha_{r n} \rho_{R D}}{\alpha_{s n} \rho_{S R}+\alpha_{r n} \rho_{R D}}\right) .
$$

Then we can highly simplify our optimization problem and update the Lloyd Algorithm by designing a new distortion function. The detailed operation is omitted here for briefness.

\section{NumERicAl RESUlts}

In this section, we present some numerical results to illustrate the performance of the new power allocation algorithm. We adopt Monte Carlo simulation for the relay system. We assume $\sigma_{r}=\sigma_{d}$ for simplicity. QPSK modulation is adopted in the simulations. $N=64$ means each OFDM symbol has 64 subcarriers. Uniform power allocation scheme and waterfilling algorithm is compared to the proposed power control strategy in Fig. 2 and Fig. 3.

It can be clearly found that the proposed power allocation policy achieves tremendous gains over the given channel. In Fig. 2, the highest curve denotes the system capacity with perfect CSI at source/relay, the lowest one denotes the system capacity of uniform power allocation strategy. We also validate the effect of the proposed scheme with limited feedback in the figures. We compare the protocol with different levels of feedback bits. We can find that only a few feedback bits is enough to achieve most of the performance gains of the perfect feedback power allocation algorithm. For an example, with 5 bit of feedback at capacity of 3.2 in Fig. 2, there is only a 1 gap to the performance limit. We can also find that further increases in the feedback bits bring degressive returns, which may implies that the feedback bits as well as the size of codebook in our model is not necessarily large.

In order to explore the effect of relay locations on the system performance, we define another parameter $\Delta=\rho_{S R}-$ $\rho_{R D}(d B)$ in the simulation, and then set $\Delta=15 \mathrm{~dB}, \Delta=-15$ $\mathrm{dB}$ and $\Delta=0 \mathrm{~dB}$. Fig. 3 demonstrate the effect of relay locations in term of BER and system capacity respectively with a fixed feedback bit level of 2 .

We can find that the proposed algorithm always outperform the uniform power allocation scheme in any kind of $\Delta$. The better channel condition $S \rightarrow R$ is, the more excellent system performance is. Besides, an interesting phenomenon is observed too. We find that the gap between the system capacities achieved by the proposed algorithm and the uniform allocation is larger when the absolute value of $\Delta$ is larger, that is, $\Delta=15 d B$ and $\Delta=-15 d B$.

\section{CONCLUSION}

In this paper, we proposed a power allocation scheme for the OFDM based relay system with limited feedback. We studied power allocation solution as the function of channel realizations with the design criteria of maximizing capacity and present an adaptation of the Lloyd algorithm to construct codebooks to quantize the optimal power allocation vectors subject to the amount of feedback. Simulations show that a negligible performance loss can be achieved with just a few feedback bits at different levels of SNR values. The new algorithm achieves a higher system capacity compared to the uniform power allocation schemes. We have further investigated the effect of the relay locations on the overall 


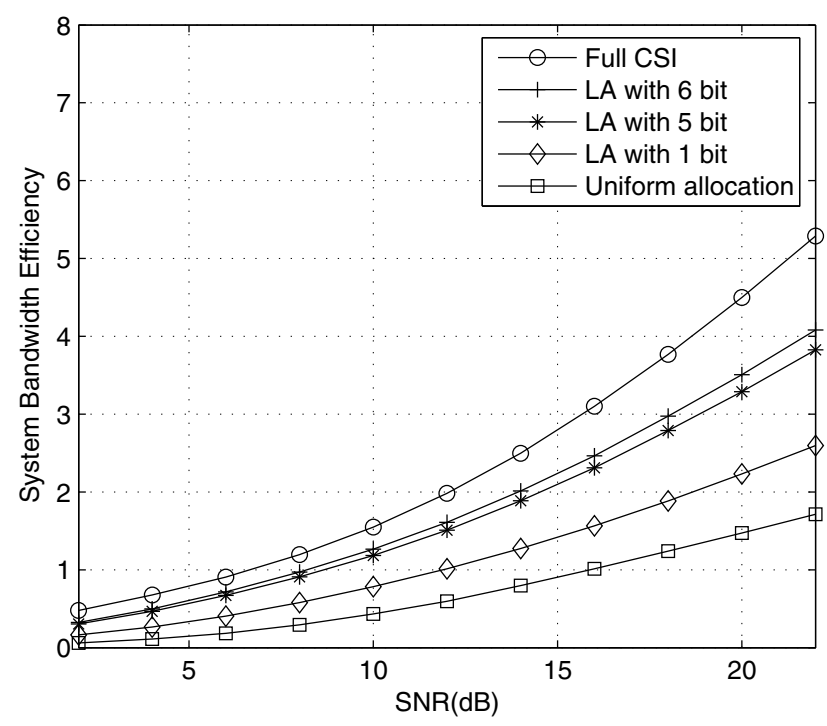

Fig. 2. System capacity versus SNR for the proposed scheme. The lowest curve denotes Constant power transmission, the highest curve is the upper bound on capacity, and the others demonstrate the effect of different feedback bits on capacity performance. LA is short for Lloyd Algorithm.

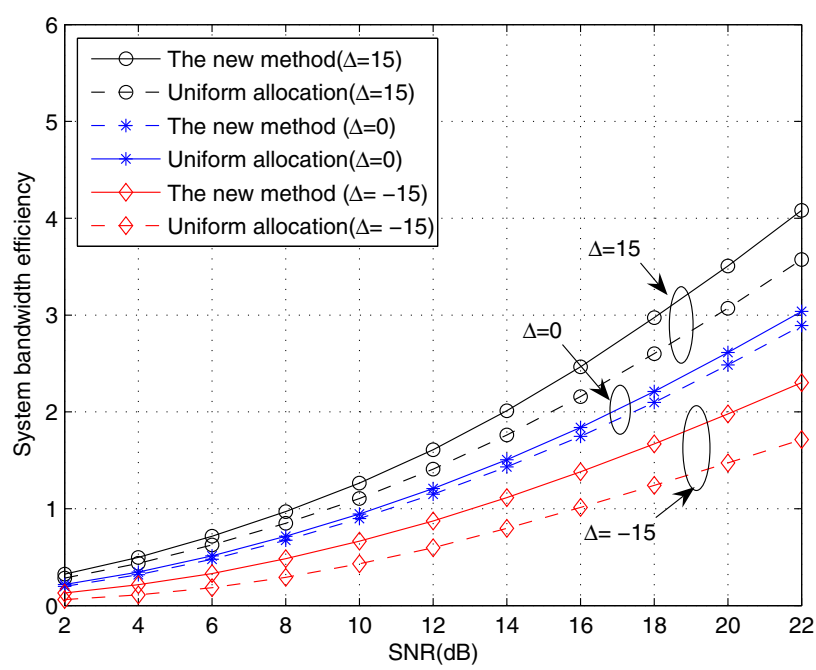

Fig. 3. System capacity versus average SNR for different setting of $\Delta$. Feedback bit is fixed on 2

performance and found that the gap between the system capacities achieved by the proposed algorithm and the uniform allocation is larger when the absolute value of $\Delta$ is larger.

\section{ACKNOWLEDGEMENT}

This work is supported by NSF China \#60972031, by SEU SKL project \#W200907, by ISN project \#ISN11-01, by Huawei Funding \#YJCB2009024WL and \#YJCB2008048WL, and by National 973 project \#2009CB824900.

\section{REFERENCES}

[1] J. N. Laneman, D. N. C. Tse, and G. W. Wornell, "Cooperative diversity in wireless networks: efficient protocols and outage behavior," IEEE Trans. Inf. Theory, vol. 50, pp. 3062-3080, Dec. 2004.

[2] R. U. Naber, H. Bolcskei, F. W. Kneubuhler, "Fading relay channels: Performance limits and spaceCtime signal design," IEEE J. Select. Areas Commun., vol. 22, no. 6, pp. 1099-1109, 2004.

[3] A. Host-Madsen and J. Zhang, "Capacity bounds and power allocation in wireless relay channel," IEEE Trans. Inf. Theory, vol. 51, pp. 20202040, Jun. 2005.

[4] C. Hoymann, K. Klagges, and M. Schinnenberg, "Multihop communication in Relay Enhanced IEEE 802.16 networks," in Proc. IEEE PIMRC'06, pp. 1-4, Sep. 2006.

[5] N. R. Van and P. Ramjee, OFDM Wireless Multimedia Communications, Boston: Artech House, 2000.

[6] Yong liu, Wen chen, "Power Allocation for the Fading Relay Channel with Limited Feedback," in Proc. IEEE ICC2010, May, 2006

[7] N. Ahmed and B. Aazhang, "Throughput Gains Using Rate and Power Control in Cooperative Relay Networks," IEEE Trans. Commun., vol. 55, no. 5, pp. 656-660, April 2003.

[8] N. Ahmed, M. A. Khojastepour, A. Sabharwal and B. Aazhang, "Outage Minimization With Limited Feedback for the fading Relay Channels," IEEE Trans. Commun., vol. 54, no. 4, pp. 659-669, April 2006.

[9] J. Zhang, Q. Zhang, C. Shao, Y. Wang, P. Zhang, and Z. Zhang, "Adaptive optimal transmit power allocation for two-hop non-regenerative wireless relay system," in Proc. IEEE VTC04, vol. 2, pp. 1213-1217, May 2004.

[10] I. Hammerstrom and A. Wittneben, "On the Optimal Power Allocation for Nonregenerative OFDM Relay Links," in Proc. IEEE ICC06, Jun. 2006.

[11] I. Hammerstrom and A. Wittneben, "Power Allocation for Amplify and Forward MIMO-OFDM Relay Links," IEEE Trans. on Wireless Commun., vol. 6, no. 8, pp. 2798-2802, Aug. 2007.

[12] W. Ying, Q. Xin-chun, and L. Bao-ling, "Power Allocation and Subcarrier Pairing Algorithm for Regenerative OFDM Relay System," in Proc. IEEE Vehicular Technology Conf. (VTC)'07, pp. 2727-2731, April 2007.

[13] Y. Ma, N. Yi, and R. Tafazolli, "Bit and Power Loading for OFDMBased Three-Node Relaying Communications," IEEE Trans. on Signal Processing, vol. 56, pp. 3236-3247, July 2008.

[14] T. T. Kim and M. Skoglund, "Diversity-multiplexing tradeoff in MIMO channels with partial CSIT," IEEE Trans. Inf. Theory, vol. 53, pp. 27432759, Aug. 2007.

[15] T. Yoo, A. Goldsmith, "Capacity and Power Allocation for Fading MIMO Channels With Channel Estimation Error," IRE Trans. Inform. Theory, vol. 52, no. 5, pp. 2203-2214, May 2006.

[16] S. Tatikonda, S. Mitter, "The Capacity of Channels With Feedback," IRE Trans. Inform. Theory, vol. 55, no. 1, pp. 323-349, Jan. 2009.

[17] C. E. Shannon, "Channels with side information at the transmitter," IBM Journal Research and Dev., vol. 2, pp. 289-293, 1958.

[18] S. Bhashyam, A. Sabharwal, and B. Aazhang, "Feedback gain in multiple antenna systems," IEEE Trans. Commun., vol. 50, no. 5, pp. 795-798, May 2002.

[19] S. Tatikonda, S. Mitter, "The Capacity of Channels With Feedback," IRE Trans. Inform. Theory, vol. 55, no. 1, pp. 323-349, Jan. 2009.

[20] J. Choi and R. W. Heath, "Interpolation based transmit beamforming for MIMO-OFDM with limited feedback," IEEE Trans. Acoust., Speech, Signal Processing, vol. 53, no. 11, pp. 4125C4135, Nov. 2005.

[21] D. J. Love and R. W. Heath, "OFDM power loading using limited feedback," IEEE Trans. Veh. Technol., vol. 54, no. 5, pp. 1773C1780, Sept. 2005.

[22] A. Gersho and R. M. Gray, "Vector Quantization and Signal Compression," Kluwer Academic Publishers, Boston, 1992.

[23] J. G. Proakis, Digital Communications, McGraw Hill, ISBN 0-07118183-0, fourth edition 2003. 